\title{
Two novel TCTN2 mutations cause Meckel-Gruber syndrome
}

\author{
Manli Zhang ${ }^{1} \cdot$ Zhijie Chang $^{2} \cdot$ Yaping Tian ${ }^{1} \cdot$ Longxia Wang $^{3} \cdot$ Yanping Lu $^{4}$
}

Received: 2 March 2020 / Accepted: 26 June 2020 / Published online: 12 July 2020

(c) The Author(s), under exclusive licence to The Japan Society of Human Genetics 2020

\begin{abstract}
Meckel-Gruber syndrome (MKS) is a clinically and genetically heterogeneous ciliopathy characterized by a triad of occipital encephalocele, polycystic kidneys, and postaxial polydactyly. Pathogenesis of MKS is related to dysfunction of primary cilia. However, reports on MKS caused by Tectonic2 (TCTN2) mutations are scanty whilst. There is no direct evidence of ciliogenesis in such MKS patients. Here, we identified two novel nonsense variants of TCTN2 (c.343G > T, p.E115*; c.1540C > T, p.Q514*) in a Chinese MKS fetus. Compared to reported TCTN2-causing MKS patients, our case represented an endocardial pad defect, which was not reported previously. We also found primary cilia protruded normally from the surface of epithelial cells in the affected fetal kidney tubules compared to controls, indicating TCTN2 is not necessary for ciliogenesis in the kidney. To our knowledge, this is the first case of MKS fetus caused by TCTN2 mutations from China.
\end{abstract}

MKS is a lethal autosomal recessive ciliopathy, showing highest incidence in Gujurat Indians resulted from consanguineous marriages [1]. The mean prevalence of MKS is 2.6 per 100,000 births in Europe [2], while it is still unclear in China. MKS is characterized by a triad of occipital encephalocele, polycystic kidneys, and postaxial polydactyly [3]. At least 21 genes are known to cause MKS $[3,4]$. Overlapping of phenotypes and genes can be observed in MKS and other ciliopathies such as Joubert

Supplementary information The online version of this article (https:// doi.org/10.1038/s10038-020-0804-0) contains supplementary material, which is available to authorized users.

$\bowtie$ Longxia Wang

13693685342@163.com

$\triangle$ Yanping Lu luyp301@163.com

1 Translational Medicine Center, Chinese PLA General Hospital, 28 Fuxing Road, 100853 Beijing, People's Republic of China

2 State Key Laboratory of Biomembrane and Membrane Biotechnology, School of Medicine, School of Life Sciences, Tsinghua University, 30 Shuangqing Road, 100084 Beijing, People's Republic of China

3 Department of Ultrasound, Chinese PLA General Hospital, 28 Fuxing Road, 100853 Beijing, People's Republic of China

4 Department of Gynaecology and Obstetrics, Chinese PLA General Hospital, 28 Fuxing Road, 100853 Beijing, People's Republic of China syndrome (JS), implying the genetic heterogeneity and pleiotropy of ciliapathies.

TCTN2 was discovered in 2006 [5]. Protein encoded by TCTN2 was regarded as membrane protein mainly located in ciliary transition zone (TZ) [6, 7]. However, TCTN2caused MKS patients were rarely reported. Associations between genotypes in TCTN2 and phenotypes in MKS remain unclear. There is also a lack of direct evidence of ciliogenesis in such MKS patients.

We encountered a healthy mother with a 13-week gestation. Early pregnancy examination results were normal until antenatal ultrasound scan at 12th-week-gestation revealed malformations in the male fetus (Fig. 1a). No family history was found. After genetic and psychological counseling, the parents decided to terminate the pregnancy with postmortem autopsy due to the fatal deformity. Chromosomal microarray analysis showed normal in this MKS fetus and its control. The two couples both agreed to voluntary donations of the fetuses. Written informed consents were obtained from the couples. The procedure above was carried out in accordance with Chinese law and regulation.

We designed a targeted capture sequencing assay to test a panel of 113 known ciliopathy genes on the affected fetus (Table S1). Putative causal variants to the reference sequence (hg19, GRCh37) were validated by Sanger sequencing. We failed in getting brain tissue in MKS fetus. Renal tissues were collected, stained with hematoxylin and eosin (HE) and scanned by electron microscopy (SEM) followed the routine method. 


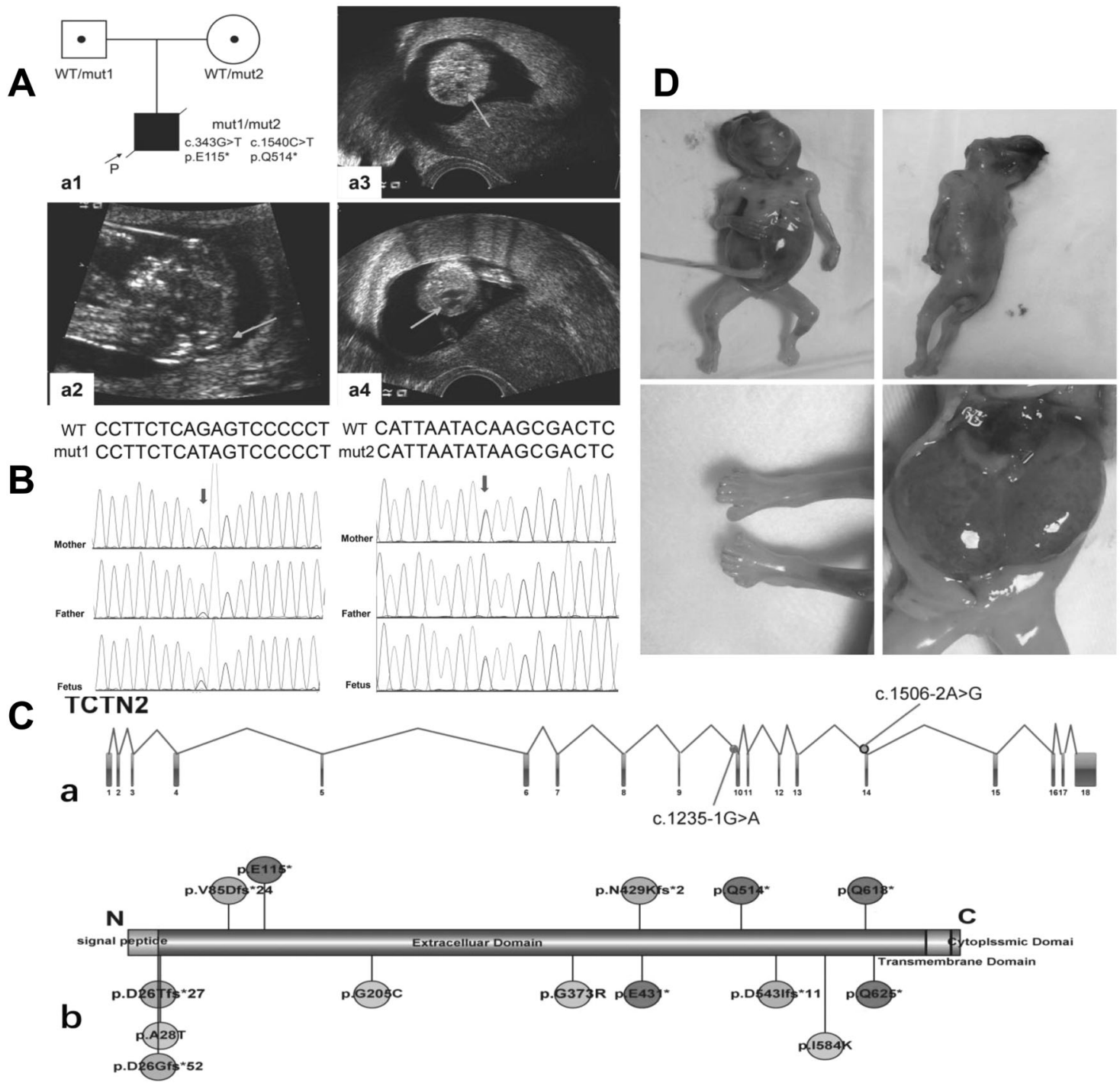

Fig. 1 Clinical features of the patient in this study and pathogenic variants reported in TCTN2-induced MKS and JS to date. a Familial pedigree and prenatal ultrasound images. In a1, the healthy father was 30 -year-old, and the mother was 28 years old. Green arrow points to occipital encephalocele of our case in a2. Ultrasound image in a3 shows bilateral polycystic kidneys in transverse section of the MKS fetal abdomen and a4 shows only one group of atrioventricular valves existing in apical four chamber view of our case. $P$ represents proband. b Two novel TCTN2 variants found in this case confirmed by Sanger sequencing which came from his parents respectively. c Sixteen

Sequencing revealed the MKS fetus carried two compound heterozygous mutations on TCTN2 (NM_024809 c.343G > T, p.E115*; c.1540C > T, p.Q514*) (Fig. 1b). The longest open reading frame of human TCTN2 gene generates a 697-amino-acid(aa) peptide. The first 25 aa is predicted as signal peptide, followed by a 643 -aa-peptide extracellular domain, a 21-aa-peptide transmembrane domain, ended with an 8-aa-peptide cytoplasmic domain (https://www.uniprot.org). The two novel TCTN2-nonsense variants both locate in extracellular domain (Fig. 1c). pathogenic variants reported in TCTN2-induced MKS and JS to date. Mutations listed above the gene or protein structure graph were from MKS patients, and the below ones were from JS patients. Two splicesite mutations in record were presented on the top of (c). Other mutaitions were presented at the bottom of (c). Red dot represents nonsence mutation. Yellow dot represents frameshift mutation. Green dot represents missence mutation. d Clinical photographs of TCTN2induced MKS fetus in this case. No chemical or environmental risk factors of malformation were discovered

Nonsense variants causing stop codon will generate truncated proteins which are presumed loss of function.

We summarized TCTN2 mutations reported in MKS and JS patients to date (Fig. 1c). All the mutations are located in the extracellular domain. In the 16 pathogenic alleles, 4 carried a missense mutation, 5 carried a small aa deletion or duplication, 2 carried a splice-site mutation, and 5 carried a nonsense mutation. It seems severe forms of mutations (nonsense and frameshift) were more likely detected in MKS patients. However, a genotype-phenotype correlation 
Two novel TCTN2 mutations cause Meckel-Gruber syndrome

1041

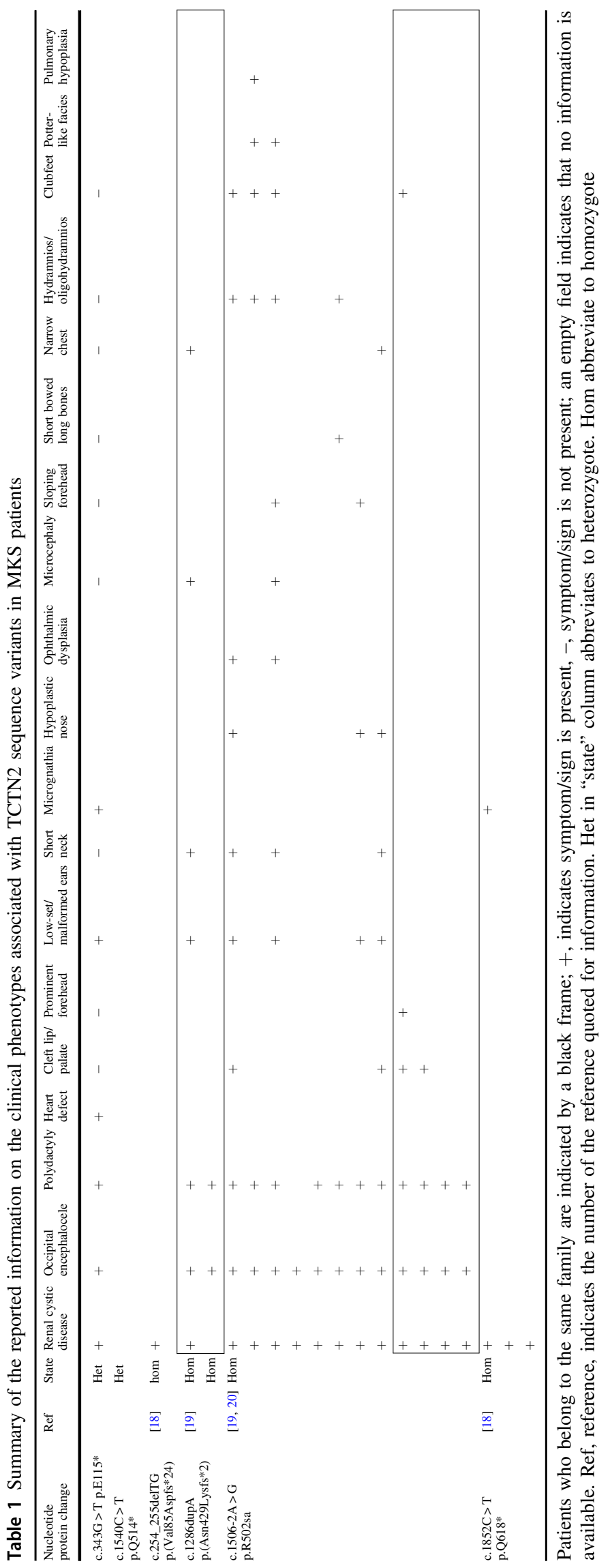

SPRINGER NATURE 
A
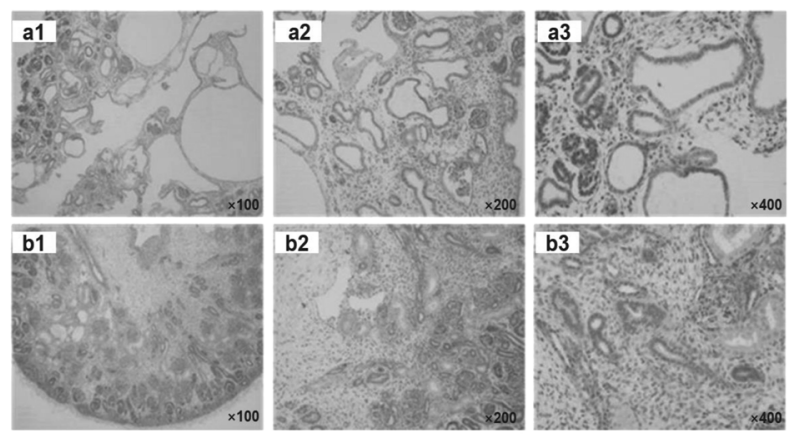

C

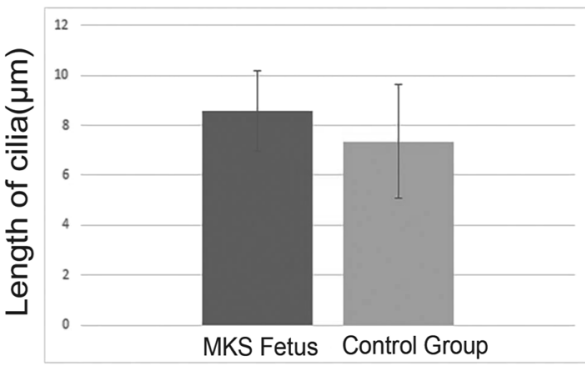

Fig. 2 Phenotypic features and cilia in kidneys of fetus with TCTN2 mutations. a Histology pattern (HE staining) of kidney in TCTN2mutated fetus (a1-a3) and the control (b1-b3). The corresponding control was from a 13-week healthy male fetus which was aborted due to his mother's severe heart disease. Magnification: $\times 100$ in $\mathrm{a} 1 / \mathrm{b} 1$, $\times 200$ in a2/b2, $\times 400$ in a3/b3.Compared with the control, kidney histology in MKS fetus shows conserved corticomedullary organization with sparsely generation of mature glomeruli. Cysts are found in

is not that obvious in TCTN2. Nonsense mutations or frameshift mutation right after signal peptide sequence in homozygous state could be found in JS patients $[8,9]$. As a membrane protein, TCTN2 protein gathered in ciliary TZ, complexed with other proteins, and is also required to localize these complex components [6, 8,10-13]. TCTN2 may function as a gatekeeper for proteins transported into and out of the cilium. The loss of transmembrane domain or change of key sites in TCTN2 probably break down the protein network in various degree, inducing MKS or JS according to response of different downstream elements.

As primary cilia present on almost each mammalian cell, resulting organs affected in MKS includes brain, kidney, liver, heart, eyes, etc. [14]. Clinical phenotypes associated with TCTN2 variants in MKS patients were listed in Table 1. Autopsy showed features in our case including low-set malformed ears, short neck, micrognathia, apart from the "triad" (Fig. 1d). In comparison, endocardial pad defect was never reported in TCTN2-induced MKS or JS. Whereas, Tctn $2^{-1-}$ mice $[6,8]$ showed ventricular septal defects, supporting the important role of TCTN2 in heart development. Unfortunately, the information about hearts of MKS patients in Table 1 is unavailable. Hence detailed clinical data from patients with TCTN2-related MKS are needed to depict whether heart defect exists. It also remains
B

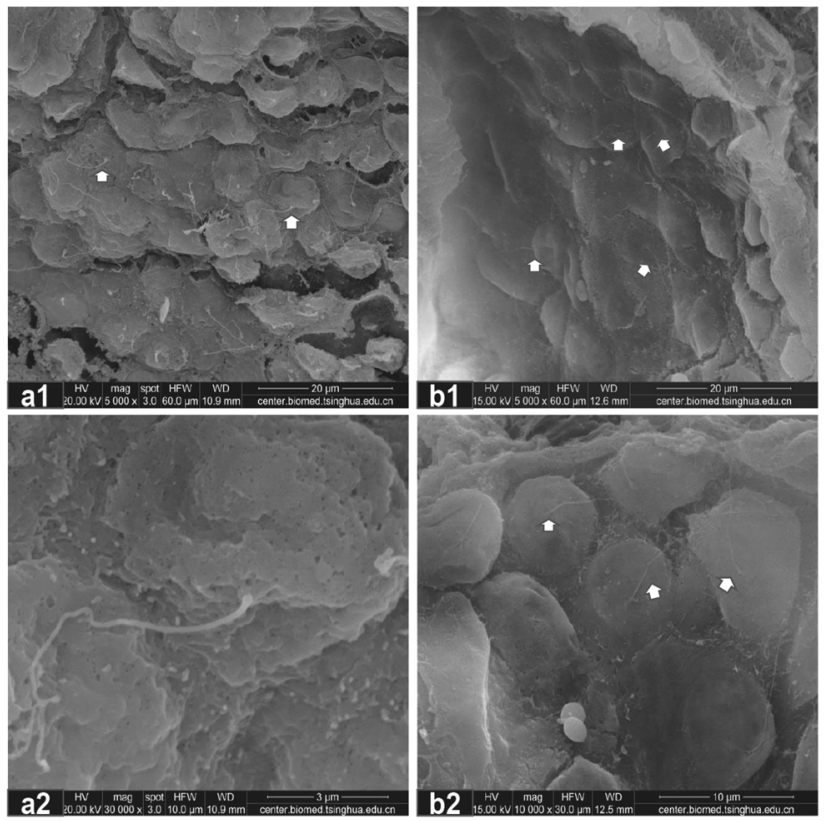

the deep cortex and medulla, and are smaller at the periphery than in the center (a1-a3). b Cilia in kidneys observed by SEM. a1 and a2: MKS fetus. b1 and b2: control. Magnification: $\times 5000$ in a1 and b1, $\times 30,000$ in $\mathrm{a} 2, \times 10,000$ in $\mathrm{b} 2$. c Cilia are measured by Image $\mathbf{J}$ in at least 12 cells in MKS fetus or control fetus. The average length of cilia in MKS fetus with TCTN2 mutations is $8.56 \pm 1.62 \mu \mathrm{m}$ while is $7.35 \pm$ $2.26 \mu \mathrm{m}$ in control group. There is no significant difference between the two groups

to be elucidated whether there are certain genotypes of TCTN2 associated with heart defect in MKS.

In Tctn $2^{-1-}$ neural tubes from mouse embryos, cilia were scarce, morphologically defective and failed to elongate axonemes, while in the limb bud mesenchyme, cilia numbers were reduced, but the remaining cilia appeared normal suggesting that TCTN2 protein was required for the ciliogenesis in a tissue-dependent manner [6]. Intriguingly, knockdown of Tctn2 in IMCD3 cells only causes modest ciliation and spheroid defects [8]. However, there were no established TCTN2 causative MKS animal model to date. And no study about primary cilia in polycystic kidney induced by TCTN2 mutations were reported. Morphological observation on the kidney by HE staining here was consistent with previous report (Fig. 2a) [15]. Then we are wondering whether it is the same in kidney of TCTN2induced MKS fetus. SEM data showed that the primary cilia protruded normally from the surface of epithelial cells in the affected fetal kidney tubules as compared to the controls (Fig. 2b). There was no significant difference between our case and controls on average length of cilia (Fig. 2c). Our data indicated that TCTN2 is not necessary for ciliogenesis in the kidney. As Tctn2 is highly expressed in embryonic brain tissues but below the limit of detection in the kidney [8], the role of TCTN2 in the development of different 
organs needs to be evaluated furtherly. Cilia were found normal in the cystic kidneys of two affected fetuses with TCTN3 mutations and TCTN3 regulates the key sonic hedgehog signaling pathway (SHH) [16]. The loss of TCTN1 does not disrupt C. elegans ciliary structure [11]. TCTN2 was also found crucial to SHH [7]. It seems pathogenesis of cystic kidney induced by tectonic member mutations is distinguished from other organs. Imbalance of signaling pathways [17], disposition of key proteins of $\mathrm{TZ}$ [6] may work together to facilitate the formation of cystic kidney.

Overall, we identified two novel TCTN2 mutations in a Chinese MKS fetus. SEM data confirmed TCTN2 is not necessary for ciliogenesis in the kidney. Downstream effectors regulated by TCTN2 on cilia in kidney development may become the targets of therapy. Further studies between correlation among TCTN2, cilia function and pathogenesis in cystic kidneys of MKS are required.

Acknowledgements We thank all patients and family members involving in this study. This work is supported by the Beijing Natural Science Foundation (7184243) and the Chinese PLA General Hospital Translational Medicine Foundation (2018TM-11) in the design, collection, analysis and the writing of the article. We thank Dr. Junnian Zhou (Beijing Institute of Radiation Medicine; South China Research Center for Stem Cell \& Regenerative Medicine, SCIB, China) for editing a draft of the paper.

\section{Compliance with ethical standards}

Conflict of interest The authors declare that they have no conflict of interest.

Ethics This paper is approved by Ethic Committee of Chinese PLA Hospital (S2019-112-01) in compliance with ethical standards.

Publisher's note Springer Nature remains neutral with regard to jurisdictional claims in published maps and institutional affiliations.

\section{References}

1. Parelkar SV, Kapadnis SP, Sanghvi BV, Joshi PB, Mundada D, Oak SN. Meckel-Gruber syndrome: a rare and lethal anomaly with review of literature. J Pediatr Neurosci. 2013;8:154-7.

2. Barisic I, Boban L, Loane M, Garne E, Wellesley D, Calzolari E, et al. Meckel-Gruber syndrome: a population-based study on prevalence, prenatal diagnosis, clinical features, and survival in Europe. Eur J Hum Genet. 2015;23:746-52.

3. Radhakrishnan P, Nayak SS, Shukla A, Lindstrand A, Girisha KM. Meckel syndrome: clinical and mutation profile in six fetuses. Clin Genet. 2019;96:560-5.
4. Parisi MA. The molecular genetics of Joubert syndrome and related ciliopathies: the challenges of genetic and phenotypic heterogeneity. Transl Sci Rare Dis. 2019;4:25-49.

5. Reiter JF, Skarnes WC. Tectonic, a novel regulator of the Hedgehog pathway required for both activation and inhibition. Genes Dev. 2006;20:22-7.

6. Garcia-Gonzalo FR, Corbit KC, Sirerol-Piquer MS, Ramaswami G, Otto EA, Noriega TR, et al. A transition zone complex regulates mammalian ciliogenesis and ciliary membrane composition. Nat Genet. 2011;43:776-84.

7. Weng RR, Yang TT, Huang CE, Chang CW, Wang WJ, Liao JC. Super-resolution imaging reveals TCTN2 depletion-induced IFT88 lumen leakage and ciliary weakening. Biophys J. 2018;115: 263-75.

8. Sang L, Miller JJ, Corbit KC, Giles RH, Brauer MJ, Otto EA, et al. Mapping the NPHP-JBTS-MKS protein network reveals ciliopathy disease genes and pathways. Cell. 2011;145:513-28.

9. Juric-Sekhar G, Adkins J, Doherty D, Hevner RF. Joubert syndrome: brain and spinal cord malformations in genotyped cases and implications for neurodevelopmental functions of primary cilia. Acta Neuropathol. 2012;123:695-709.

10. Dowdle WE, Robinson JF, Kneist A, Sirerol-Piquer MS, Frints SG, Corbit KC, et al. Disruption of a ciliary B9 protein complex causes Meckel syndrome. Am J Hum Genet. 2011;89:94-110.

11. Yee LE, Garcia-Gonzalo FR, Bowie RV, Li C, Kennedy JK, Ashrafi $\mathrm{K}$, et al. Conserved genetic interactions between ciliopathy complexes cooperatively support ciliogenesis and ciliary signaling. PLoS Genet. 2015;11:e1005627.

12. Chih B, Liu P, Chinn Y, Chalouni C, Komuves LG, Hass PE, et al. A ciliopathy complex at the transition zone protects the cilia as a privileged membrane domain. Nat Cell Biol. 2011;14:61-72.

13. Shi X, Garcia G III, Van De Weghe JC, McGorty R, Pazour GJ, Doherty D, et al. Super-resolution microscopy reveals that disruption of ciliary transition-zone architecture causes Joubert syndrome. Nat Cell Biol. 2017;19:1178-88.

14. Youn YH, Han YG. Primary cilia in brain development and diseases. Am J Pathol. 2018;188:11-22.

15. Mougou-Zerelli S, Thomas S, Szenker E, Audollent S, Elkhartoufi $\mathrm{N}$, Babarit C, et al. CC2D2A mutations in Meckel and Joubert syndromes indicate a genotype-phenotype correlation. Hum Mutat. 2009;30:1574-82.

16. Thomas S, Legendre M, Saunier S, Bessieres B, Alby C, Bonniere $\mathrm{M}$, et al. TCTN3 mutations cause Mohr-Majewski syndrome. Am J Hum Genet. 2012;91:372-8.

17. Srivastava S, Molinari E, Raman S, Sayer JA. Many genes-one disease? Genetics of nephronophthisis (NPHP) and NPHPassociated disorders. Front Pediatr. 2017;5:287.

18. Al-Hamed MH, Kurdi W, Alsahan N, Alabdullah Z, Abudraz R, Tulbah M, et al. Genetic spectrum of Saudi Arabian patients with antenatal cystic kidney disease and ciliopathy phenotypes using a targeted renal gene panel. J Med Genet. 2016;53:338-47.

19. Shaheen R, Szymanska K, Basu B, Patel N, Ewida N, Faqeih E, et al. Characterizing the morbid genome of ciliopathies. Genome Biol. 2016;17:242.

20. Shaheen R, Faqeih E, Seidahmed MZ, Sunker A, Alali FE, AlQahtani K, et al. A TCTN2 mutation defines a novel Meckel Gruber syndrome locus. Hum Mutat. 2011;32:573-8. 